\title{
Impact of Oral Sensory Motor Stimulation on Feeding Performance, Length of Hospital Stay, and Weight Gain of Preterm Infants in NICU
}

\author{
Sharife Younesian ${ }^{1}$; Fariba Yadegari ${ }^{1, *}$; Farin Soleimani ${ }^{2}$ \\ ${ }^{1}$ Department of Speech Therapy, School of Rehabilitation, University of Social Welfare and Rehabilitation Sciences, Tehran, IR Iran \\ 2 Pediatric Neurorehabilitation Research Center, University of Social Welfare and Rehabilitation Sciences, Tehran, IR Iran \\ *Corresponding Author: Fariba Yadegari, Department of Speech Therapy, School of Rehabilitation, University of Social Welfare and Rehabilitation Sciences, Tehran, IR Iran. \\ Tel/Fax: +98-2122180043, E-mail: Faribayadegari@yahoo.com
}

Received: July 16, 2013; Revised: September 7, 2014; Accepted: March 20, 2015

\begin{abstract}
Background: One of the limiting factors for early hospital discharge in preterm infants is their inability to feed sufficiently to obtain consistent weight gain. Therefore, feeding difficulty is one of the most significant issues with which a preterm infant is faced.

Objectives: The purpose of this study was to examine the effect of oral sensory motor stimulation on feeding performance, length of hospital stay, and weight gain in preterm infants at 30 - 32 weeks of gestational age.

Patients and Methods: Premature infants $(n=20)$ were randomly assigned to experimental and control groups. The experimental group received oral sensory motor stimulation of the oral structures (15 minutes / day) for 10 successive days, while these stimulations were not offered to the control group. Days elapsed to achieve oral feeding, length of hospital stay, and weight gain in the two groups were assessed. Results: Transition to oral feeding was acquired significantly earlier in the infants in the experimental group than in the controls: 13 and 26 days, respectively $(\mathrm{P}<0.001)$. Likewise, the length of hospitalization was significantly shorter in the experimental group than in the control group: 32 days and 38 days, correspondingly $(\mathrm{P}<0.05)$. The two groups showed no significant difference in terms of weight gain in the first, second, third, and fourth weeks of birth: first week: 100 vs. 110; second week: 99 vs. 111; third week: 120 vs. 135 ; and fourth week: 129 vs. 140.

Conclusions: The present research revealed that the number of days to reach oral feeding in our preterm babies was decreased by oral motor stimulation, which in turn conferred earlier hospital discharge.
\end{abstract}

Keywords: Preterm Infant; Hospital; Weight Gain

\section{Background}

The last century has witnessed a considerable rise in the survival of young preterm infants with feeding difficulties thanks to the advances in their care. Sucking is regarded as the best way to feed newborns; however, the sucking skills of preterm infants become mature between weeks 32 and 34 (1). Oral feeding difficulties are almost common in preterm infants due to problems in their cardiorespiratory and central nervous systems as well as the incomplete development of their oral structures (2).

Coordinated suck-swallow-breath pattern is a significant aspect of a successful feeding. Under 32 weeks of gestational age, most preterm infants are not able to have an independent oral feeding and they are fed by tubes (1). Tube feeding throughout hospital stay affects the ability of infants to obtain full oral feeding, which may cause considerable feeding disorders and aversive or hypersensitive responses to being stroked around or in the mouth $(3,4)$. Indeed, the ability of new-born babies to have a full oral feeding is necessary for their discharge from the hospital $(5,6)$, where a variety of multidisciplinary team members support preterm infants to feed orally and discharge earlier (7). The effects of early oral motor difficul- ties on breast /bottle feeding and the length of hospital stay underline the significance of the need to improve the normal development of the oral motor structures (3). Various intervention techniques are used by nutritionists to bolster the oral feeding performance of premature infants. One of the widely used strategies is the sensory oral motor input, which comprises cheek/chin support and touch and visual, vestibular, and auditory stimulations (8-10). The effectiveness of some interventions has been previously proved. For example, cheek/chin support during the feeding time raises the intake volume (8) and an auditory-tactile-visual-vestibular program speeds up the transition to independent oral feeding, reducing hospital stay (11).

Gaebler and Hanzlik (8) reported that infants receiving an oral pre-feeding stimulation exhibited better performance on the Neonatal Oral Motor Assessment Scale, better weight gain, and shorter hospital stay in all relevant studies. There is an evidence-based systematic review of oral motor interventions in preterm infants carried out by Arvedson et al. (12), who reported that 7 of 12 studies evaluating the effects of non-nutritive sucking (NNS) on

Copyright ( 2015, Iranian Red Crescent Medical Journal. This is an open-access article distributed under the terms of the Creative Commons Attribution-NonCommercial 4.0 International License (http://creativecommons.org/licenses/by-nc/4.0/) which permits copy and redistribute the material just in noncommercial usages, provided the original work is properly cited. 
feeding parameters showed that the preterm infants achieved full oral feeding earlier, although there were no significant differences regarding the other criteria such as weight gain and intake volume. Two of the 12 studies examined just the effects of touch therapy on feeding performance. The results of these studies, however, were not sufficient, and the authors noted that there was limited evidence to support the effect of touch therapy without NNS. Three of the 12 studies examined the effects of both touch therapy and NNS on the efficiency of feeding in preterm infants: the experimental groups reached independent oral feeding earlier than the control groups (12). Therefore, substantial evidence implies that sensory oral motor input to the oral structures or oral motor stimulation during NNS exerts significant effects on the performance of oral feeding if given before or while feeding by mouth in premature infants with medical stability (3).

\section{Objectives}

The purpose of the present study was to examine the effects of an early oral motor stimulation program on the performance of oral feeding in preterm infants. The oral motor program was commenced prior to the introduction of oral feeding since the aim was to improve oral motor skills before the probable development of oral feeding disorders.

\section{Patients and Methods}

Participants were chosen from the Neonatal Intensive Care Unit (NICU) at Valie Asr Hospital in Imam Khomeini Hospital Complex, Tehran, Iran. This referral treatment center is a governmental general hospital, including a 20bed NICU and several wards and clinics.

Using mean and SD values from a previous study by Fucile et al. (3) with a power of $80 \%$ and confidence level of $95 \%$, we reached a sample size of 10 for each group. From 30 subjects being examined initially, 20 preterm neonates (10 boys and 10 girls) who met our inclusion-exclusion criteria were randomly assigned to the experimental group (10 newborns) and the control group (10 newborns). The enrolled infants were: 1) born between 30 and 32 weeks' gestational age, which was set in accordance with the date of last menstruation and the first-trimester ultrasound; 2) appropriate for gestational age; 3 ) fed by tube; and 4) without chronic medical complications such as bronchopulmonary dysplasia, intraventricular hemorrhage grades 3 and 4, periventricular leukomalacia, necrotizing enterocolitis, and congenital anomalies examined by a neonatologist as the exclusion criteria.

This research was approved by the Research Ethics Committee of the University of Social Welfare and Rehabilitation Sciences, Tehran, Iran (Code = USWR.REC.1393.103). The parents were free to agree or disagree with the inclusion of their babies into the experimental group and to withdraw cooperation whenever they deemed fit, and they were given reassurances as to the confidentiality of their newborns' medical condition. Parental consent was gained before the infants were entered into the study.

\subsection{Procedure}

In this experimental research with convenience sampling, a random assignment was performed using a simple randomization method. The study began on July 2011 and lasted for 5 months. An oral sensory motor stimulation program was given to the experimental group, and the infants in the control group received no stimulation except routine nursery. The oral stimulation was not administered as routine in this hospital. Interventions were started before the outset of oral feeding and were applied once per day for 10 sequential days, 20 to 40 minutes before the initiation of tube feeding. The commencement and advancement of oral feeding was assigned to the attending physician. Both nurses and physicians were blinded to group assignment.

If the infants were not fully stable 45 minutes before the intervention, the program was not implemented. The program was also discontinued if the infants had medical instability and/or had an oxygen instauration and apnea/bradycardia throughout the intervention. All the infants in both groups were monitored from the beginning of the intervention to hospital discharge. Breast feeding was the routine in this hospital. The types and times of feeding per day were recorded. Independent oral diet, length of hospital stay, and weight gain were the dependent variables of this research. Independent oral diet for an inborn was begun at the discretion of a physician, who was blinded to group assignment. After the start of the independent oral diet of a baby, the number of the independent oral diets for each baby in each day was recorded by the nurses. The first time an infant achieved 8 oral feedings per day for 2 consecutive days was defined as the time to gain full oral feeding.

The weight gain of each infant was measured and recorded using a digital balance. A digital scale sensitive to one $\mathrm{g}$ (Seca 334, Mobile digital baby scale, CE 0123) was used daily for weight measurement. This equipment is calibrated every 3 months by the company. The babies were weighed by the same nurse every day at $7 \mathrm{a} . \mathrm{m}$. without clothes and diapers and before feeding.

The practitioners who measured the newborns' weight were blinded to their assigned group and hospital discharge time. The length of hospital stay was calculated from the newborns' day of birth.

\subsection{Interventions}

This program was in accordance with the Beckman principles $(1,3,10,13,14)$. The program was performed once a day for 10 successive days, 20 to 40 minutes before feeding by tube. Oral stimulation can enhance the performance of infants' oral feeding if the procedure is carried out before feeding time (15). The oral sensory motor stimulation program comprised a 15-minute stimula- 
tion program, whose first 12 minutes included stroking the newborns' cheeks, lips, gums, and tongue, and whose last 3 minutes included the newborns' sucking on an index finger of the speech therapist, who was trained by the researchers. Latex gloves were used for touch therapies. The intervention group was controlled by cardiorespiratory monitoring and pulse oximetry before, during, and after the intervention.

\subsection{Outcome Measure}

The number of days necessary for preterm infants to transit from tube feeding to oral feeding was considered as the time to reach oral feeding. The fulfillment of the entire feeding without any occurrence of oxygen unsaturation and/ or apnea/bradycardia implied a successful feeding. The commencement and advancement of oral feeding was in accordance with the unit's written routine and was similar for both groups. The number of days which the infants spent from birth to hospital discharge was considered the length of hospital stay. Weight gain from the first to fourth weeks of birth was determined.

The following covariates were taken into account: the infants' behavioral state at the beginning and end of the feeding time via the preterm infant's behavioral scale (16); bradycardia; apnea; and oxygen desaturation throughout oral feeding.

\subsection{Statistical Analysis}

As the data were normally distributed (KolmogorovSmirnov), parametric tests were recruited for the analyses. Yet, given our small sample size, the results were rechecked using non-parametric tests, and as the results confirmed the parametric probes, they are not reported here. A t-Student test was employed to specify the influence of the oral sensory motor stimulation on the time of the achievement of the specified number of oral feedings during a day (i.e. one, 4 , and 8 successful oral feedings per day) and length of hospitalization. In order to assess the within-subject effect, at time points of one to 4 and 8 independent oral feedings per day, a repeated measures ANOVA was applied. The level of significance was 0.05 . SPSS (version 16) was used for the analyses.

\section{Results}

The baseline characteristics were compared between the two groups (Table 1). Both groups received all the covariates equally $(\mathrm{P}>0.05)$. There were no significant differences in terms of gestational age and birth weight between the two groups.

Table 2 demonstrates the ages at which the oral feeding of the experimental and control groups occurred. The infants in the experimental group achieved one $(\mathrm{P}<0.001)$, $4(\mathrm{P}<0.001)$, and $8(\mathrm{P}<0.001)$ successful oral feedings significantly in fewer days of life than the controls. Therefore, the experimental group attained oral feeding milestones faster than the controls. In fact, the experimental group reached an oral feeding ( 8 oral feedings per day) approximately 2 weeks earlier than the controls.

From the whole 100 oral motor stimulation sessions that were managed, there were 8 delayed or halted sessions. Four sessions were offered with delay because the infants were distracted 20 minutes before the onset of the intervention, 2 sessions were implemented with delay due to medical instability, and 2 sessions were cancelled because the infants had an episode of bradycardia. The experimental and control groups were discharged after $32 \pm 6$ and $38 \pm 2$ days, correspondingly. The infants in the experimental group acquired an early hospital discharge compared with the controls insofar as they were discharged approximately one week earlier.

A repeated measures ANOVA with a Greenhouse-Geisser correction determined that the mean weight of the case group differed significantly between the time points $(\mathrm{F}[1.937,17.434]=44.892 ; \mathrm{P}<0.0001)$. Post-hoc tests using the Bonferroni correction revealed that touch therapy did not evoke a change in weight from pre-intervention to one oral feeding $(1590 \pm 314.2 \mathrm{~g}$ vs. $1523 \pm 323.9 \mathrm{~g}$, respectively), which was not statistically significant $(\mathrm{P}=0.183)$. However, the weight change from one to 4 oral feedings a day was significant $(1523 \pm 323.9$ g vs. $1573 \pm 319.2$ g, respectively; $\mathrm{P}=0.001)$. The weight change from 4 to 8 oral feedings per day was also significant $(1573 \pm 319.2 \mathrm{~g}$ vs. $1624 \pm 327.7 \mathrm{~g}$, correspondingly; $\mathrm{P}=0.002)$. Finally, the weight change from 8 oral feedings a day to the time of discharge from the NICU was also significant (1624 \pm 327.7 g vs. $1877 \pm 234.7$ g, respectively; $\mathrm{P}=0.001$ ) (Figure 1 ).

\begin{tabular}{|c|c|c|c|c|}
\hline Group & Mean \pm SD & $\mathbf{t}$ & df & Significance Level \\
\hline Birth weight & & 0.279 & 18 & 0.783 \\
\hline Case ( 5 boys and 5 girls) & $1590 \pm 0.52$ & & & \\
\hline Control ( 5 boys and 5 girls) & $1548 \pm 0.52$ & & & \\
\hline Gestational age & & 0.878 & 18 & 0.391 \\
\hline Case & $31.20 \pm 0.78$ & & & \\
\hline Control & $30.90 \pm 0.73$ & & & \\
\hline
\end{tabular}


Table 2. Comparison of the Mean Age of the Experimental and Control Newborns for One, 4 , and 8 Independent Oral Feedings per $\operatorname{Day}(\mathrm{n}=10)^{\mathrm{a}}$

\begin{tabular}{|c|c|c|c|c|}
\hline Group & Mean \pm SD & $\mathbf{t}$ & df & Significance Level \\
\hline D.O.L.1 O.F. & & -5.342 & 18 & 0.000 \\
\hline Case & $6.50 \pm 2.59$ & & & \\
\hline Control & $13.10 \pm 2.92$ & & & \\
\hline G.A.1 O.F. & & -1.826 & 18 & 0.084 \\
\hline Case & $31.84 \pm 0.78$ & & & \\
\hline Control & $32.52 \pm 0.87$ & & & \\
\hline D.O.L.4 O.F. & & -6.118 & 18 & 0.000 \\
\hline Case & $9.80 \pm 3.42$ & & & \\
\hline Control & $19.70 \pm 3.80$ & & & \\
\hline G.A.4 O.F. & & -2.953 & 18 & 0.009 \\
\hline Case & $32.34 \pm 0.72$ & & & \\
\hline Control & $33.51 \pm 1.02$ & & & \\
\hline D.O.L.8 O.F. & & -6.716 & 18 & 0.000 \\
\hline Case & $13.20 \pm 4.31$ & & & \\
\hline Control & $26.90 \pm 4.79$ & & & \\
\hline G.A.8 O.F. & & -3.758 & 17 & 0.002 \\
\hline Case & $32.84 \pm 0.81$ & & & \\
\hline Control & $34.47 \pm 1.04$ & & & \\
\hline D.O.L. discharge & & -5.233 & 18 & 0.000 \\
\hline Case & $27.90 \pm 6.15$ & & & \\
\hline Control & $38.80 \pm 2.34$ & & & \\
\hline
\end{tabular}

a Abbreviations: D.O.L, days of life (days); G.A., gestational age (weeks); and O.F., oral feeding per day.

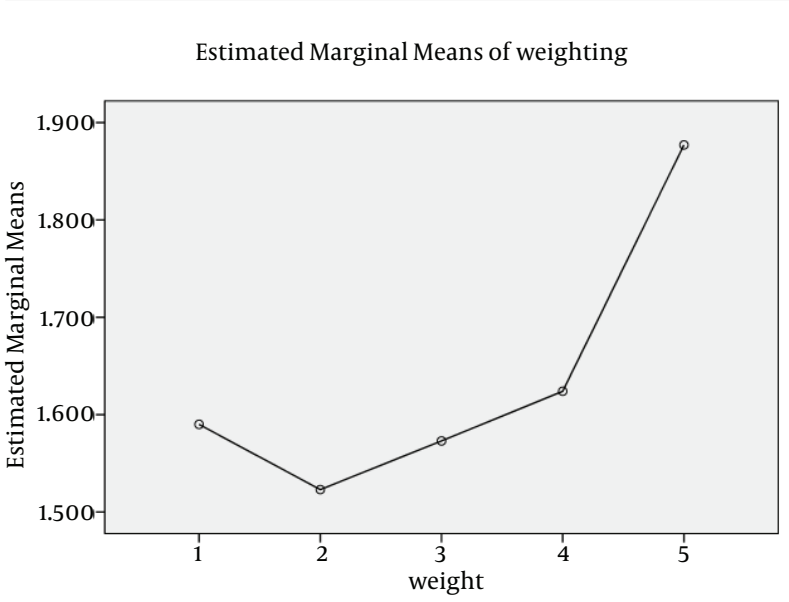

Figure 1. Estimated Weight Gain of Newborns in Case Group Through Oral Feeding

A repeated measures ANOVA with a Greenhouse-Geisser correction determined that the mean weight of the control group was also significantly different between the time points $(\mathrm{F}[1.577,14.193]=41.953 ; \mathrm{P}<0.001)$. Post-hoc tests using the Bonferroni correction revealed a change in birth weight to one oral feeding (1548 $\pm 112.7 \mathrm{~g}$ vs. $1489 \pm 102.5 \mathrm{~g}$, respectively), which was not statistically significant ( $\mathrm{P}=$ 0.082). Nonetheless, the weight change from one to 4 oral feedings per day was significant $(1489 \pm 102.5 \mathrm{~g}$ vs. $1556 \pm$ $103.2 \mathrm{~g}$, correspondingly; $\mathrm{P}=0.001)$. The weight change from 4 to 8 oral feedings per day was also significant (1556 \pm 103.2 g vs. $1650 \pm 103.2$ g, respectively; $P=0.001$ ). Finally, the weight change from 8 to the time of discharge from the NICU was also significant $(1650 \pm 103.2 \mathrm{~g}$ vs. $1843 \pm 84.85 \mathrm{~g}$, correspondingly; $\mathrm{P}=0.002$ ) (Figure 2 ). As is evident from the results, both groups gained weight significantly, but the case group reached this milestone earlier. This is shown in a comparison of the discharge time between the two groups. Although the t-Student test proved the time of discharge was not significantly different between the two groups, the mean discharge time of the experimental group was evidently shorter than that of the controls (Table 3). Because of the low sample size, a bootstrap was performed, the results of which were similar to the t-test (Table 4). Error bar plots are illustrated in Figure3. 


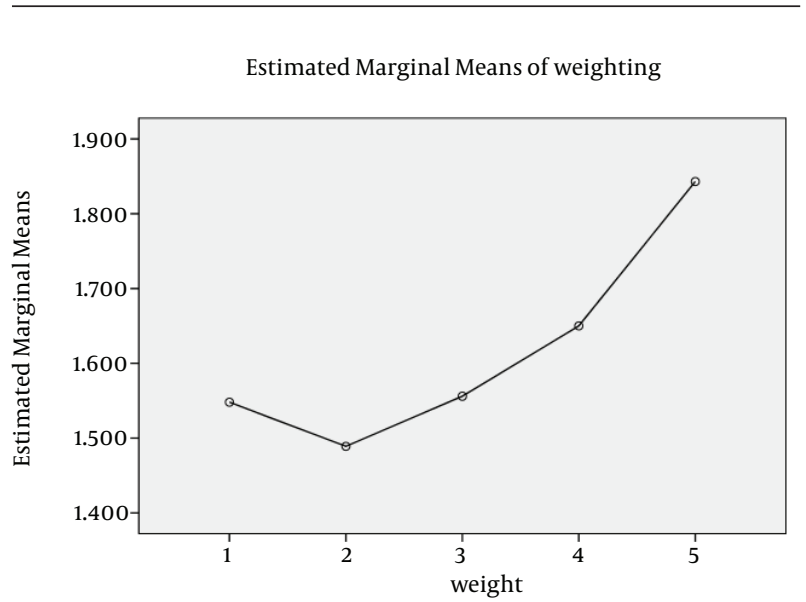

Figure 2. Estimated Weight Gain of Newborns in Control Group Through Oral Feeding

Table 3. Comparison of the Mean Time of Discharge from the NICU between the Experimental and Control Groups $(n=10)$

\begin{tabular}{lcccc}
\hline & Mean \pm SD & t & df & $\begin{array}{c}\text { Significance } \\
\text { Level }\end{array}$ \\
\hline Group & & -0.934 & 18 & 0.362 \\
Case & $5.78 \pm 0.75$ & & & \\
Control & $6.22 \pm 1.27$ & & & \\
\hline
\end{tabular}

Table 4. Results of Bootstrapping for the Difference of Discharge Time between the Experimental and Control Groups

\begin{tabular}{lccc}
\hline \multirow{2}{*}{ Standard Error } & Sig. (2-tailed) & \multicolumn{2}{c}{ 95\% CI } \\
\cline { 3 - 4 } & & Lower & Upper \\
\hline $\mathbf{0 . 4 5 8}$ & 0.368 & -1.28490 & 0.45474 \\
\hline
\end{tabular}

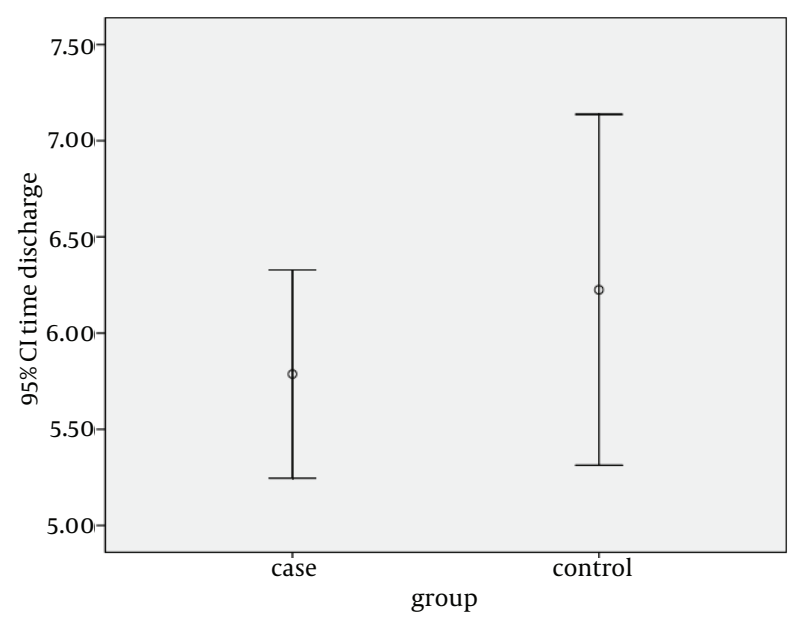

Figure 3. Summary Bar Plot of Mean Time of Discharge (95\% Confidence Interval) for Cases and Controls

\section{Discussion}

The length of the hospital stay of infants born under the age of 30 weeks' gestational age is approximately between 11 and 12 weeks (4). Because the rate of infants who survive under the age of 30 weeks' gestational age is increasing and there is plenty of evidence indicating that most preterm infants are born with feeding disorders (2), an assessment of whether or not early oral sensory motor stimulation can be effective on the feeding performance of preterm infants is vitally important.

The results of the current study show that the oral feeding performance of our preterm infants was modified by using the sensory oral motor stimulation accompanied by NNS and that it led to early hospital discharge. In this study, the preterm infants who received the program achieved independent oral feeding earlier than the control group. In addition, the infants in the experimental group were discharged earlier than the controls. Both groups were similar with respect to such baseline characteristics as birth weight and gestational age at birth as well as oxygen requirement. In addition, the study groups were alike in that they had no chronic medical complications. Thus, that the experimental group achieved earlier oral feeding does not mean that its newborns were healthier and/or more mature than the controls.

The infants in the experimental group achieved oral feeding 2 weeks earlier than the controls. There are some convincing reasons why the infants who received intervention achieved oral feeding earlier than their counterparts. The first part of the program, which included some stroking, may have strengthened the oral motor structures which have a significant role in adequate sucking. Moreover, NNS, the next part of the oral motor stimulation program, may have provided the infants with the experience of sucking. Furthermore, it is likely that the oral motor stimulations offered in the current study expedited the maturation of the central and peripheral neural structures, thereby improving the infants' sucking skills and suck-swallow- breath coordination $(13,16)$.

In the current study, the infants in the experimental group were discharged one week earlier. Owing to the fact that the experimental infants gained oral feeding significantly earlier than the controls, it was expected that their hospitalization would be shorter than the controls. In a study by Fucile et al. (3), there was no substantial difference between the experimental and control groups in relation to the length of hospital stay, which may be due to the absence of a universally practiced discharge standard. Bragelien et al. (14) did not find any connections between oral stimulation and early oral feeding. However, it is worthy of note that they used different stimulation techniques. Although another study cited in Rocha et al. (1) used only the NNS technique, they observed a significant difference between the two groups in terms of the length of hospital stay.

Lau et al. (17) employed a nonnutritive oral motor thera- 
py (NNOMT) or infant massage therapy (IMT) in preterm infants and noted that these interventions had a direct positive impact on speeding up the maturation of their infants' oral feeding performances. In our study, we did not seek to determine whether or not this intervention is more beneficial than NNS alone; further studies are needed to shed sufficient light on this question. Also, it is noteworthy that as we utilized a convenience method of sampling with a relatively small sample size, our results should be interpreted cautiously and this may limit the external validity of our results. Indeed, future studies are required to determine whether our intervention period was appropriate and sufficient.

According to the findings of the current study, early sensory oral motor stimulation with NNS in preterm infants may be effective to expedite oral feeding and hospital discharge. Therefore, we can conclude that a touch therapy program may confer a statistically significant weight gain for premature babies at much shorter intervals, which per se leads to a shorter hospital stay.

\section{Acknowledgements}

We would like to express our appreciation to the following for their contributions to this project: Valie Asr Hospital for its valuable cooperation in carrying out this study; University of Social Welfare and Rehabilitation Sciences for its financial support; and the nursery team in the NICU of Tehran Valiasr Hospital.

\section{Authors' Contributions}

Sharife Younesian: developed the original idea and the intervention, collected data and analyzed data, wrote the manuscript. Fariba Yadegari: contributed to the development of the intervention, and prepared and wrote the manuscript, analyzed data. Farin Soleimani: developed the intervention, prepared the manuscript, and collected data.

\section{Funding/Support}

We recieved a grant from University of Social Welfare and Rehabilitation Sciences, Tehran, IR Iran.

\section{References}

1. Rocha AD, Moreira ME, Pimenta HP, Ramos JR, Lucena SL. A randomized study of the efficacy of sensory-motor-oral stimulation and non-nutritive sucking in very low birthweight infant. Early Hum Dev. 2007;83(6):385-8.

2. Bu'Lock F, Woolridge MW, Baum JD. Development of co-ordination of sucking, swallowing and breathing: ultrasound study of term and preterm infants. Dev Med Child Neurol. 1990;32(8):669-78.

3. Fucile S, Gisel E, Lau C. Oral stimulation accelerates the transition from tube to oral feeding in preterm infants. $J$ Pediatr 2002;141(2):230-6.

4. Schanler RJ, Shulman RJ, Lau C. Feeding strategies for premature infants: beneficial outcomes of feeding fortified human milk versus preterm formula. Pediatrics. 1999;103(6 Pt 1):1150-7.

5. Lessen BS. Effect of the premature infant oral motor intervention on feeding progression and length of stay in preterm infants. Adv Neonatal Care. 2011;11(2):129-39.

6. Zimmerman EA, Barlow SM. The Complexity of Transitioning to Oral Feeds in Preterm Infants. Perspect Speech Sci Orofac Disord. 2009;19(1):52.

7. Greene Z, O'Donnell CPF, Walshe M. Oral stimulation techniques in preterm infants - International research challenges.J Neonatal Nurs. 2013;19(4):168-74.

8. Gaebler CP, Hanzlik JR. The effects of a prefeeding stimulation program on preterm infants. Am J Occup Ther. 1996;50(3):184-92.

9. Hill AS, Kurkowski TB, Garcia J. Oral support measures used in feeding the preterm infant. Nurs Res. 2000;49(1):2-10.

10. Fucile S, Gisel EG, Lau C. Effect of an oral stimulation program on sucking skill maturation of preterm infants. Dev Med Child Neurol. 2005;47(3):158-62.

11. White-Traut RC, Nelson MN, Silvestri JM, Vasan U, Littau S Meleedy-Rey P, et al. Effect of auditory, tactile, visual, and vestibular intervention on length of stay, alertness, and feeding progression in preterm infants. Dev Med Child Neurol. 2002;44(2):91-7.

12. Arvedson J, Clark H, Lazarus C, Schooling T, Frymark T. Evidencebased systematic review: effects of oral motor interventions on feeding and swallowing in preterm infants. Am J Speech Lang Pathol. 2010;19(4):321-40.

13. Pimenta HP, Moreira ME, Rocha AD, Gomes JSC, Pinto LW, Lucena SL. Effects of non-nutritive sucking and oral stimulation on breastfeeding rates for preterm, low birth weight infants: a randomized clinical trial. J Pediatr (Rio J). 2008;84(5):423-7.

14. Bragelien R, Rokke W, Markestad T. Stimulation of sucking and swallowing to promote oral feeding in premature infants. Acta Paediatr. 2007;96(10):1430-2.

15. Lynn S, Robin P. Feeding and Swallowing Disorders in Infancy. First edition. A division of The Psychological corporation. Arizona; 1992.

16. Lau C, Sheena HR, Shulman RJ, Schanler RJ. Oral feeding in low birth weight infants. J Pediatr. 1997;130(4):561-9.

17. Lau C, Fucile S, Gisel EG. Impact of nonnutritive oral motor stimulation and infant massage therapy on oral feeding skills of preterm infants. J Neonatal Perinatal Med. 2012;5(4):311-7. 